\title{
Occlusion cues contribute to orientation judgments of occlusion-defined contours
}

\author{
P. DE WEERD, E. VANDENBUSSCHE, and G. A. ORBAN \\ Katholieke Universiteit te Leuven, Campus Gasthuisberg, Leuven, Belgium
}

\begin{abstract}
Occlusion cues defining a contour in a 2-D stimulus pattern were shown to contribute to the accuracy of orientation judgments of that contour. The stimulus pattern was altered so that the occlusion cues became ambiguous, by introducing a textured background suggesting transparency of the stimulus pattern. Orientation judgments then became significantly less accurate. This finding shows that occlusion cues in 2-D patterns can be behaviorally relevant, in addition to generating the subjective percept commonly known as an illusory contour. The disruptive effect of the textured background on orientation judgments remained when no texture elements were present in the vicinity of the contour. This suggests that the generation of occlusion-defined contours relies as much on an evaluation of the surfaces at either side of the contour as being opaque as it does on local encoding of occlusion cues close to the contour. Finally, orientation sensitivity measured with contours defined by other than occlusion cues was not altered after the introduction of a textured background.
\end{abstract}

An illusory contour can be defined as a boundary that crosses regions of homogeneous physical stimulation (Schumann, 1900). Illusory contours are often generated by a particular arrangement of elements such as line ends or corners. An explanation of illusory contours that has gained ground over the last decade holds that the visual system interprets such elements as occlusion cues. In other words, the visual system gives a three-dimensional (3-D) interpretation to patterns in which 2-D occlusion cues suggest that one surface is occluding another (Baumgartner, von der Heydt, \& Peterhans, 1984; Brady \& Grimson, 1981; Peterhans, von der Heydt, \& Baumgartner, 1984). This hypothesis has been invoked to explain a number of illusions, both in static (Kanisza, 1955) and in kinetic (Anderson \& Cortese, 1989; Bruno \& Bertamini, 1990) displays. Furthermore, several authors have insisted that the processes responsible for creating a 3-D impression in 2$D$ illusory contour patterns are identical to the processes that recover depth from occluding surfaces in the reallife visual environment (Brady \& Grimson, 1981; Baumgartner et al., 1984; Peterhans et al., 1984; Peterhans, von der Heydt, \& Baumgartner, 1986; von der Heydt, 1987). Hence, the perception of illusory contours due to

\footnotetext{
We wish to thank R. Vogels for helpful discussions during the course of the experiments, and P. Kayenbergh and G. Meulemans for expert technical assistance. We are also grateful to $A$. Lueschow and L. Chelazzi for valuable comments on earlier versions of the manuscript. Most of all, we thank the subjects, H. Kinable, G. Caudron, I. Leenen, and R. Van Den Broek, for their participation in the experiments. For the subjects, participation in the experiments was a partial fulfillment of requirements for the MA in psychology. P.D.W. was a junior research fellow at Leuven and is now a visiting research associate at the National Institute of Mental Health. Correspondence should be sent to G. A. Orban, Laboratorium voor Neuro- en Psychofysiologie, Katholieke Universiteit te Leuven, Herestraat 49, B 3000 Leuven, Belgium.
}

occlusion cues might reflect a highly relevant process in vision. The occlusion hypothesis does not exclude the contribution of other factors, such as assimilation and dissimilation, to the perception of illusory contours (Halpern, 1981, 1983; Parks, 1984; Pritchard \& Warms, 1983).

A number of investigators have tried to quantify the subjective qualities of visual illusions. For instance, a variety of measures have been devised to quantify the impression of depth in illusory-contour-generating stimulus patterns (Coren, 1972; Coren \& Girgus, 1978; Gregory, 1972; Halpern, 1981; Porac, 1978; Shank \& Walker, 1989). In the present study, instead of quantifying the subjective strength of the illusion caused by potential occlusion cues, we investigated whether the accuracy of the perception of a contour induced by line ends depends on the interpretation of the line ends as occlusion cues. In particular, if it is correct that the interpretation of contourinducing elements as occlusion cues contributes to contour perception, one might expect the accuracy of contour perception to decrease if additional cues in the stimulus pattern were to contradict the interpretation of the inducing elements as occlusion cues.

The necessity for the visual system to interpret inducing line ends as occlusion cues can be removed by presenting the stimulus pattern on a textured background (Reynolds, 1981; Rock \& Anson, 1979). This makes the surfaces at either side of the contour transparent, which is difficult to reconcile with the idea of two opaque occluding surfaces. Rather, the elements surrounding the contour are perceived as disparate and floating in front of the background, instead of belonging to solid surfaces. The choice of a texture to suggest a background behind the stimulus pattern is in accord with the work of Rubin (1958), who pointed out that a background must be formless, suggesting a substance rather than a thing, which is essentially 
what a texture is. The impression of the texture's being a background can be further enhanced by making the texture elements less bright than the elements that define the contour (Metelli, 1974a, 1974b).

Thus, we compared the accuracy of contour perception in a condition in which the contour-inducing elements were presented on a homogeneous background with the accuracy in a condition in which inducing elements were presented on a textured background. The accuracy of contour perception was measured in a contour orientation discrimination task. Human beings are very sensitive to the orientation of occlusion-defined contours (Vogels \& Orban, 1987), just as they are to the orientation of other types of contours (e.g., Mustillo, Francis, Oross, Fox, \& Orban, 1990; Orban, Vandenbussche, \& Vogels, 1984). Therefore, it is desirable to use orientation thresholds as a tool for detecting possible changes in the accuracy of contour perception after the introduction of a textured background.

\section{GENERAL METHOD}

\section{Subjects}

Four subjects (H.K., G.C., R.V.D.B., and I.L.) participated in the experiments, in addition to one of the authors (P.D.W.). H.K., P.D.W., and G.C. were trained psychophysical observers. R.V.D.B. and I.L. had received only limited training prior to the experiments, and were naive with regard to their underlying hypothesis. P.D.W., H.K., and G.C. are nearsighted, with their vision corrected to normal.

\section{Apparatus and Stimulus}

The stimuli were presented on a $720 \times 1,440$ pixel monochrome Atris screen, having a refresh rate of $75 \mathrm{~Hz}$. The viewing distance was $175 \mathrm{~cm}$. During the experiments the subject's head was stabilized by means of a headframe and chinrest. The experiments were carried out in the dark.

The standard stimulus consisted of a set of four circle halves shifted in phase relative to a set of four companion circle halves (Figures 1A-1B). The circle halves were white $\left(12.2 \mathrm{~cd} / \mathrm{m}^{2}\right)$ on a black background $\left(0.03 \mathrm{~cd} / \mathrm{m}^{2}\right)$. The circle width was $0.03^{\circ}$. The standard spacing between neighboring endpoints (line ends) was $0.26^{\circ}$, and the standard contour length was $3.92^{\circ}$. The fixation spot was a small circle, $0.06^{\circ}$ in diameter.

In a number of experimental conditions, the stimuli were presented on a background of texture elements. The density of the texture elements was 9.35 per square visual degree. These textures were made either of line segments (line texture, Figure $1 \mathrm{C}$ ) or of annuli (annulus texture, Figure 1D). The line elements were $0.29^{\circ}$ long and $0.03^{\circ}$ wide. The annulus elements had the same surface as did the line elements. The luminance of the texture elements was $6.01 \mathrm{~cd} / \mathrm{m}^{2}$, which is about half the luminance of the inducing semicircles.

The stimulus exposure time was $300 \mathrm{msec}$. The stimuli were followed by a brief $(50-\mathrm{msec})$ masking texture that was identical to the background textures, except that it consisted of $50 \%$ annuli and $50 \%$ lines and that the luminance of the texture elements was $12.02 \mathrm{~cd} / \mathrm{m}^{2}$. The mask was used to avoid a significant contribution of afterimages to the orientation judgments.

\section{Procedure}

Orientation discrimination was measured using a standard increment, increment standard (SIIS) design (Vogels \& Orban, 1986). The standard stimulus (S) in our experiments was always horizontal; the increment stimulus (I) was rotated clockwise from horizontal In the SIIS design, the two stimuli are presented sequentially, the order being randomly determined. Subjects were instructed to press the right-hand key after the SI sequence and the left-hand key after the IS sequence. The interstimulus and intertrial intervals were $1,000 \mathrm{msec}$ each. The subjects had to respond within $600 \mathrm{msec}$ after the mask following the second stimulus. Correct responses were followed by a tone. The use of a horizontal standard stimulus allowed us to use two naive observers in our experiments (R.V.D.B. and I.L.) without extensive pretraining (see Vogels \& Orban, 1985, 1987).

Just noticeable differences (JNDs) in orientation were determined with an $84 \%$ correct Wetherill and Levitt (1965) staircase procedure. Following this procedure, the orientation difference between the two sequentially presented stimuli was decreased after four consecutive correct responses and increased after a single incorrect one. Changes in orientation difference were obtained by multiplying or dividing by a factor of 1.25 . The measurements were stopped after 10 reversal points. JNDs were calculated as the geometrical mean of the last 6 reversal points.

The center of each stimulus was presented at random in one of four locations, each of them $1.04^{\circ}$ away from the fixation spot. Two of these locations were situated on a left oblique axis through the fixation spot (left above and right below relative to the fixation point, Figures 1A-1B). Analogously, the two other stimulus center locations were located on a right oblique axis (right above and left below, Figures 1C-1D). In addition to the randomization of stimulus position, also contour length and the spacing between endpoints were randomized in a range of $\pm 10 \%$ of the standard length and spacing. We also randomized the sign of the phase shift between the two sets of inducing semicircles. These randomizations reduced the possibility that subjects would solve the orientation discriminations by using the position of isolated endpoints, or by using the global orientation available in the outer border of the stimulus pattern (compare Figures 1C and 1D) instead of contour orientation.

\section{EXPERIMENT 1}

\section{Method}

In this preliminary experiment, JNDs were measured in a condition in which the stimulus pattern was presented on a black background (Figures 1A-1B), and one in which the stimulus was presented on a background texture made of line elements (Figure $1 \mathrm{C}$ ). Three consecutive measurements per background condition were obtained for 3 subjects (H.K., G.C., and P.D.W.). The order of background conditions was randomized over subjects. This experiment was run twice, once before introducing the stimulus randomizations described in the General Method section, and once after introducing those randomizations. Hence, in the former case, the center of the stimulus pattern always coincided with the fixation point; and length, endpoint spacing, and shift direction were fixed.

\section{Results and Discussion}

Figure 2 compares the JNDs in orientation obtained in the two background conditions for each subject separately, before (Figure 2A), and after (Figure 2B) introduction of the stimulus randomizations. Open symbols refer to thresholds determined using a black background. Solid symbols correspond to data obtained with the textured background. In general, JNDs in orientation were elevated $(U=48, p<.01)$ when the stimulus pattern was presented on a texture background (solid symbols). This effect will be referred to as the texture background effect. A clear exception was H.K. (Figure 2A). However, since a fixed stimulus pattern was used, this subject might have 
BACKGROUND

CONDITION 1

A

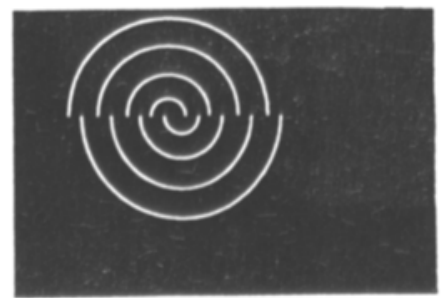

BACKGROUND

C

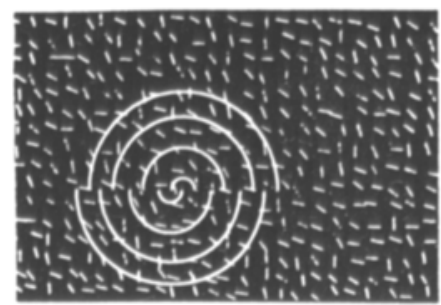

BACKGROUND

$E$

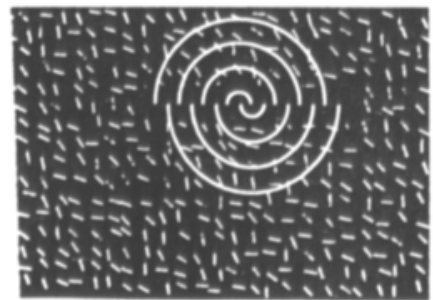

BACKGROUND

G

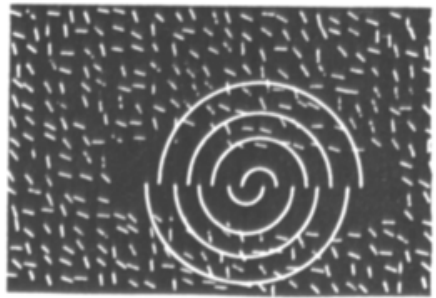

B

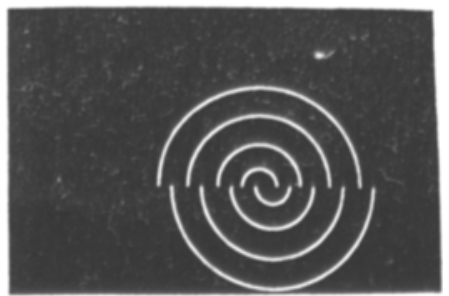

CONDITION 2

$\mathrm{D}$

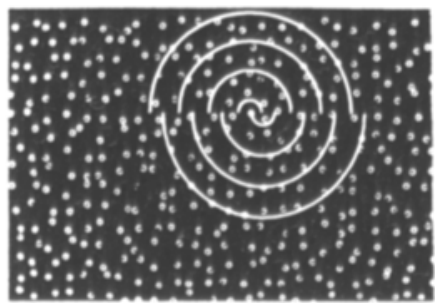

CONDITION 3

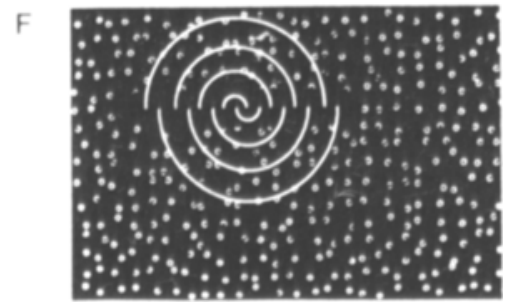

CONDITION 4

$H$

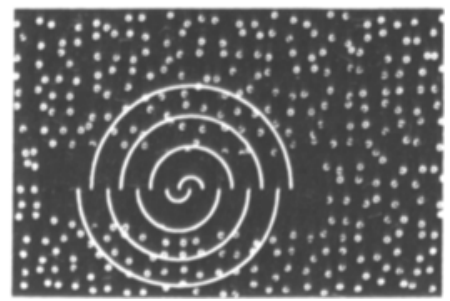

Figure 1. Collection of representative stimuli used in Experiments 1 and 2. The four rows correspond to four possible background conditions. In the first row (A, B), stimuli are presented on a black background (Condition 1$)$. In the second row $(C, D)$, the stimulus pattern is superimposed on a background texture (Condition 2$)$. In the third (E, F) and fourth (G, $\mathrm{H})$ background conditions, texture elements are erased within a specified window around the contour (see Experiment 2 for details). In the left column (A, C, E, G), the background textures are made of line elements. In the right column, annulus elements are used to make the background textures. The figure also illustrates the randomizations in stimulus position, length, endpoint distance, and direction of phase shift described in the General Method section. The fixation spot (not shown) was always presented in the middle of the screen.

used a cue other than contour orientation, and therefore the effect of introduction of the texture condition-thought to affect contour perception-might have been obscured. This hypothesis is confirmed by the general increase of H.K.'s thresholds and the emergence of the texture background effect when the randomized stimulus was used (Figure 2B). The findings for Subject H.K. illustrate the importance of randomizing the necessary stimulus param- eters if one wants to study contour orientation judgments. Altogether, the results support the hypothesis that reducing the strength of the occlusion cues at an occlusion border also reduces the accuracy of orientation judgments of this border.

The next experiment was designed to answer the following additional questions. First, the line texture offers additional line ends near the contour, which could con- 

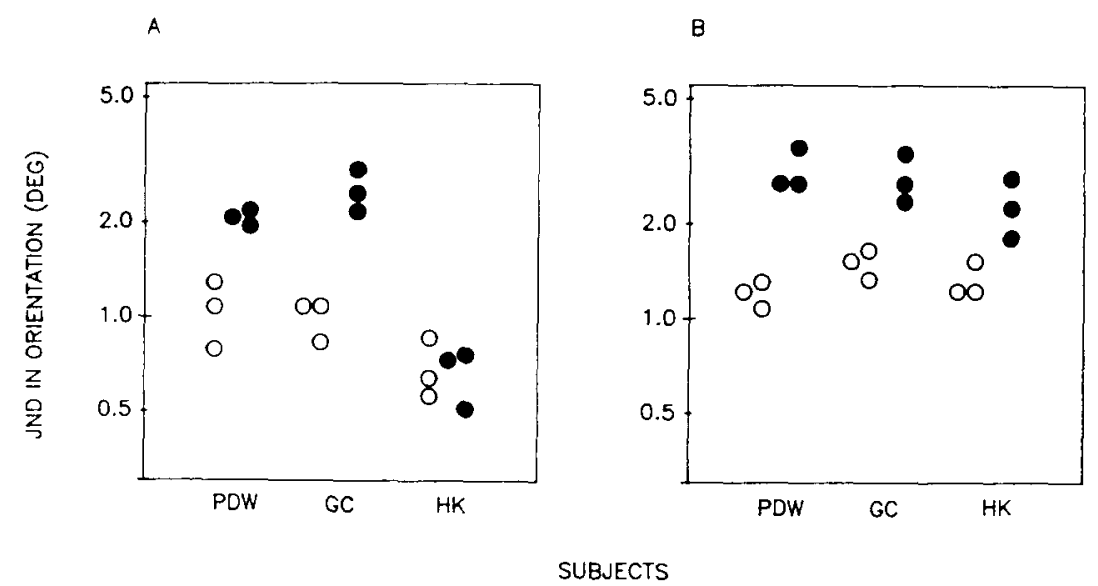

Figure 2. JNDs in orientation for 3 subjects (P.D.W., G.C., and H.K.) before (A) and after (B) introduction of the various stimulus parameter randomizations described in the General Method section. Each symbol represents a single JND. Open symbols refer to JNDs obtained with patterns presented on a black background. Solid symbols refer to JNDs measured with the use of a textured background made of line elements.

found any mechanism extracting the contour on the basis of local analysis of line ends. In addition, the line texture elements sometimes attach to the line ends belonging to the illusory contour pattern, rendering even less obvious which ends are to be considered in the process of contour extraction. If the increase in thresholds in the texture condition depended on a local mechanism, the effect should vanish if texture elements in the immediate vicinity of the contour are removed. In addition, if the presence of additional line ends is critical for the texture background effect to occur, using the annulus texture as a background might be expected to reduce the effect because the local mechanism is supposed to be orientation selective. Finally, the texture background effect might be a consequence of the global contrast reduction between the inducing elements $\left(12.2 \mathrm{~cd} / \mathrm{m}^{2}\right)$ and the global texture background. Indeed, global luminance of the textured backgrounds used was $0.245 \mathrm{~cd} / \mathrm{m}^{2}$ compared to $0.03 \mathrm{~cd} / \mathrm{m}^{2}$ for the black background. This comes down to a reduction in contrast from 2.61 to $1.69(\log \Delta \mathrm{I} / \mathrm{I})$, which might be sufficient to explain the texture background effect.

\section{EXPERIMENT 2}

\section{Method}

JNDs in orientation were determined for 4 subjects (G.C., I.L., R.V.D.B., P.D.W.) in 16 different conditions. In each condition, a single threshold was determined for each subject. Four different background conditions were used. In Background Condition 1, the stimulus was presented on a black background (Figures 1A-1B). In the second condition, a texture was used as a background (Figures $1 C-1 D$ ). In the third condition, texture elements were wiped out in a rectangular window extending $0.67^{\circ}$ above and below the contour (Figures $1 \mathrm{E}-1 \mathrm{~F}$ ). In the fourth condition (Figures $1 \mathrm{G}-1 \mathrm{H}$ ), this window extended $1.33^{\circ}$ above and below the contour. The orientation of the window in the texture was randomized in an angle of $\pm 1.5^{\circ}$ around the contour's orientation. Control experiments showed that the results obtained did not depend on whether or not the window orientation was correlated with contour orientation. Furthermore, JNDs in orientation were measured in two different luminance conditions. In the low-luminance condition, the luminance of the circular inducing elements $\left(1.49 \mathrm{~cd} / \mathrm{m}^{2}\right)$ was chosen so that the contrast with the black $0.03 \mathrm{~cd} / \mathrm{m}^{2}$ background matched the contrast of the standard $12.2 \mathrm{~cd} / \mathrm{m}^{2}$ inducing elements with the 0.245 $\mathrm{cd} / \mathrm{m}^{2}$ texture background $[\log (\Delta \mathrm{I} / \mathrm{I})=1.69]$. Finally, all conditions were repeated twice, once in combination with a line texture as a background and once in combination with an annulus texture as a background. In summary, three factors were manipulated: the type of background (4 background conditions), the luminance of the inducing elements (2 luminance conditions), and the type of texture element constituting the background textures ( 2 texture types).

\section{Results and Discussion}

Figure 3 shows the results obtained in this experiment. In all nine graphs in this figure, JNDs in orientation are plotted as a function of background condition. The background conditions are indicated by the numbers on the $x$-axes. The solid and open symbols refer to the high- and the low-luminance conditions, respectively. The texture backgrounds graphed in Figure 3A were made of line elements, whereas those in Figure 3B were made of annuli. In Figures $3 A$ and $3 B$, the graphs represent the results from the individual subjects, and Figure $3 \mathrm{C}$ shows the results averaged over subjects and texture types. A randomized factorial block analysis of variance (ANOVA) revealed the background condition to be the only significant factor $[F(3,45)=29.43, p<.01]$. The increase of the JNDs in Background Condition 2 compared with those for Condition 1 in the high-luminance condition (solid symbols) confirmed the results of Experiment 1. The luminance factor was not significant $[F(1,45)=0.87$, n.s. $]$. Moreover, JNDs in the low- and high-luminance conditions at Background 1 were not different from each other $[F(1,45)=0.07$, n.s.]. Since the contrast of the inducing elements with the black background in the low-luminance condition (Figure 3: Condition 1, open symbols) equalled 
A

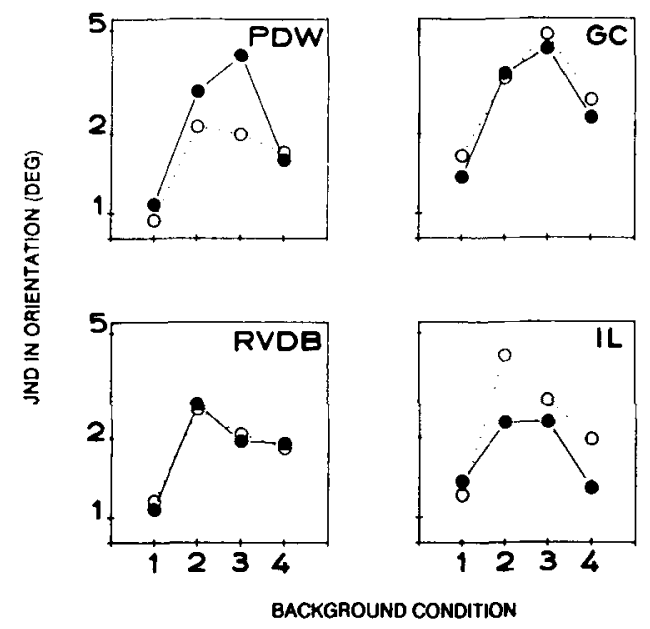

$\mathbf{B}$
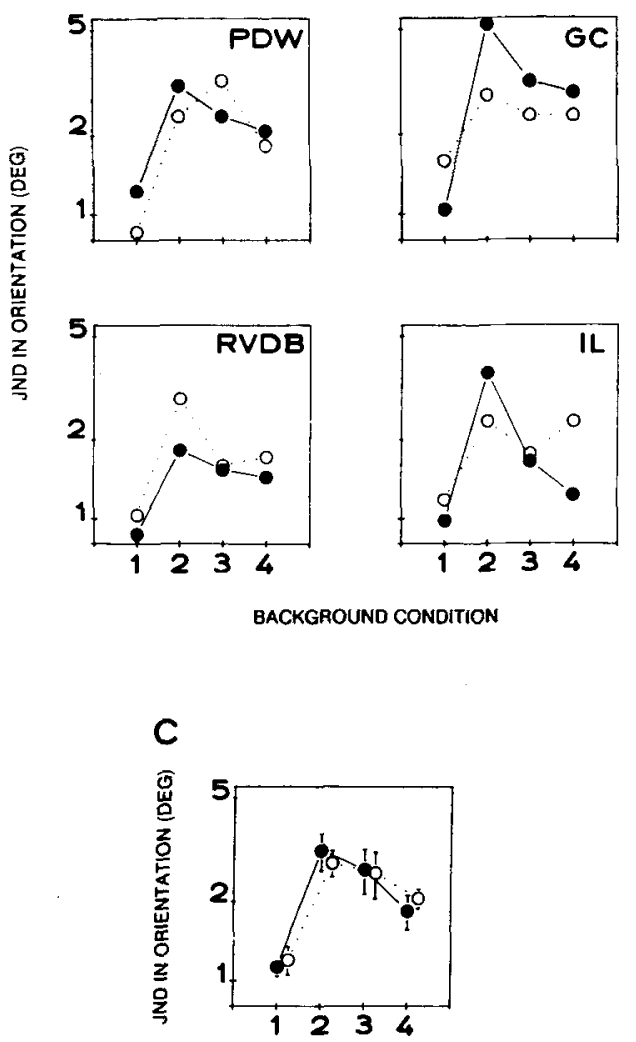

BACKGROUNO CONOITION

Figure 3. JNDs in orientation plotted as a function of background condition for 4 subjects (P.D.W., G.C., R.V.D.B., and I.L.) in the line texture (A) and the annulus texture (B) conditions. (C) JNDs averaged over subjects and texture type conditions. Background Condition 1 refers to a black background in Figures 1A-1B, Condition 2 refers to the texture backgrounds shown in Figures 1C-1D, Condition 3 refers to Figures $1 E-1 F$, and Condition 4 refers to the texture backgrounds in Figures 1G-1H. Solid symbols refer to JNDs determined in the high-luminance condition. Open symbols refer to the low-luminance condition. Error bars in $\mathrm{C}$ are standard errors. the contrast of the inducing elements with the textured background in the high-luminance condition (Figure 3: Condition 2, solid symbols), the increase of the thresholds in the latter condition was not a consequence of the reduced contrast caused by the textured background. In addition, the effect of the texture background remained despite removal of the texture elements in the immediate vicinity of the contour (Background Condition 3). In fact, there was no significant difference in thresholds between Background Conditions 2 and $3[F(1,45)=2.99$, n.s. $]$. In Background Condition 4, however, JNDs were smaller than in Condition $2[F(1,45)=24.28, p<.01]$, but they remained significantly elevated in comparison with those in Condition $1[F(1,45)=14.33, p<.01]$. These results suggest that the texture background effect was not merely due to a local interference with the encoding of the endpoints defining the contour. Furthermore, the texture background effect did not depend on the type of texture elements that the textures were made of $[F(1,45)=$ 1.69, n.s.]. This shows that the disruptive effect of the texture background was not specific for the type of texture elements. Finally, it is also worth mentioning that the same results were obtained at a $600-\mathrm{msec}$ stimulus exposure time (Subjects G.C. and P.D.W.).

From the summary of the results in Figure $3 \mathrm{C}$, obtained after averaging over subjects and texture type conditions, one can estimate the average magnitude of the texture background effect. In the high-luminance condition, JNDs increased by a factor of 2.76 from $1.13^{\circ}$ in Background Condition 1 to $3.12^{\circ}$ in Background 2. The texture background effect remained strong in Background Condition 3 $\left(\mathrm{JND}=2.65^{\circ}\right)$ and weakened in Background $4(\mathrm{JND}=$ $\left.1.84^{\circ}\right)$. As discussed previously, the results in the lowluminance condition were very similar: JNDs were $1.20^{\circ}$, $2.80^{\circ}, 2.57^{\circ}$, and $2.06^{\circ}$ in Background Conditions $1-4$, respectively.

The present results show that the texture background effect is not an artifact of contrast reduction between inducing elements and global background. In addition, this effect is not merely due to a failure in the local processing of endpoints, since it remains when texture elements are removed from the immediate vicinity of the inducing endpoints. A more global factor must be involved. We suggest that the rise in orientation thresholds after the introduction of a background texture is due at least partly to a reduction of the strength of the occlusion cues that define the contour. To further strengthen this point, we compared the effect of the background texture among three more sorts of contours, one of them defined by strong occlusion cues, the two others defined by cues other than occlusion cues. If the background texture effect really were due to the diminishing of occlusion cues, this effect should be weak or absent for contours that are not defined on the basis of occlusion.

\section{EXPERIMENT 3}

\section{Method}

For 2 subjects, three different contours were used to determine JNDs in orientation. First, a stimulus pattern was created that con- 
A

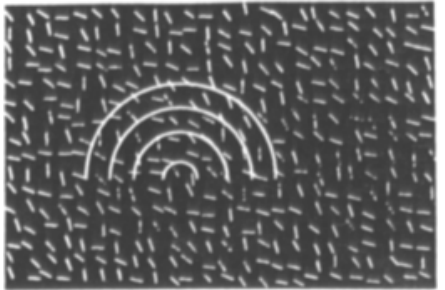

C

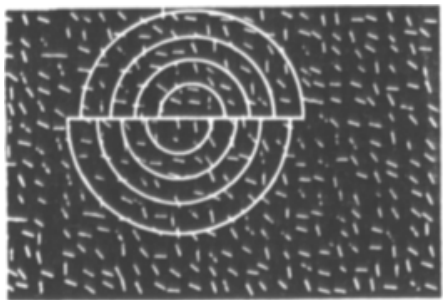

$\mathrm{E}$

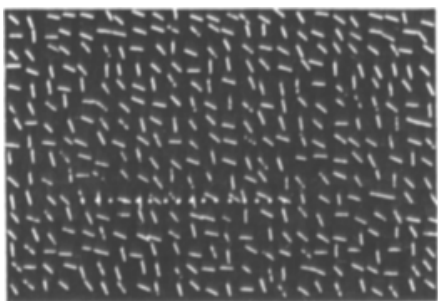

B

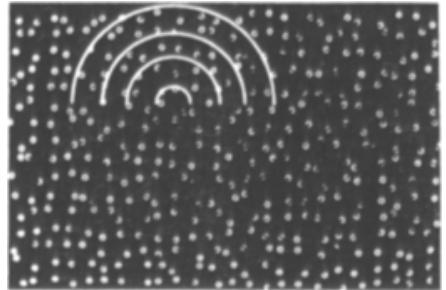

$\mathrm{D}$

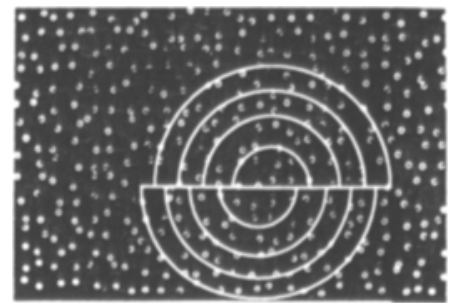

$\mathrm{F}$

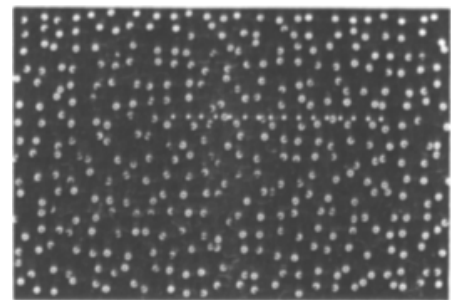

Figure 4. Three additional contour types used in Experiment 3. In one case, the standard pattern was reduced to the upper half $(A, B)$. In the second case, a line was drawn coinciding with the occlusion-defined contour $(C, D)$. Third, a dotted line was used, the dots corresponding to the endpoints of the inducing circle halves in the standard pattern (E, F). The left column ( $\mathrm{A}, \mathrm{C}, \mathrm{E})$ shows the stimuli on the line texture background. In the right column, the stimuli are presented on the annulus background.

sisted of a single set of semicircles only (Figures 4A-4B). The subjects were always presented with the upper set of circle halves. Second, a straight line was put into the stimulus pattern, connecting the endpoints of the circle halves (the width and luminance of the line and circle halves were equal; Figures 4C-4D). Third, only the endpoints of the inducing circles were shown (Figures $4 \mathrm{E}-4 \mathrm{~F}$ ). The first pattern contained strong occlusion cues, occlusion cues were not necessary to derive a contour from the second stimulus pattern, and occlusion cues were absent in the third pattern. JNDs in orientation were measured by using each of these three contour types in the four background conditions used in the previous experiment; either line textures or annulus textures were the texture backgrounds. A single threshold was determined in each condition for each subject.

\section{Results and Discussion}

Figure 5 shows the results, together with a replot of the results obtained with the standard pattern in Experiment 2 (solid dots). The latter results were included in the statistical analysis of the data. Figure $5 \mathrm{~A}$ shows the results in the line texture condition for 2 subjects. Figure $5 \mathrm{~B}$ presents the data obtained in the annulus texture condition. JNDs in orientation again proved to be unaffected by the type of texture used as a background
$[F(1,31)=0.10$, n.s. $]$. There were significant main effects of contour type $[F(3,31)=97.90, p<.01]$ and background condition $[F(3,31)=27.560, p<.01]$. The effect of background condition, however, was clearly different for the two contours that were mainly based on occlusion cues (complete illusory contour pattern and half version, plotted as solid dots and solid, inverted triangles, respectively) in comparison with the two non-occlusioncue contours (straight line and dotted line, shown as open squares and open, upright triangles, respectively). For the black background (Background Condition 1), there was no significant difference in thresholds between the two pairs of contour types $[F(1,31)=1.496$, n.s. $]$. In contrast, the JNDs obtained with occlusion-defined contours were strongly affected by the background manipulation, whereas JNDs measured with non-occlusion-defined contours were not. The difference in JNDs between occlusionand non-occlusion-defined contours was significant at the .01 level for Background Conditions 2, 3, and $4[F(1,31)=$ $143.38,116.06$, and 40.07 , respectively].

The results are summarized in Figure $5 \mathrm{C}$. The average JNDs in Background Condition 1 varied between 0.67 
A
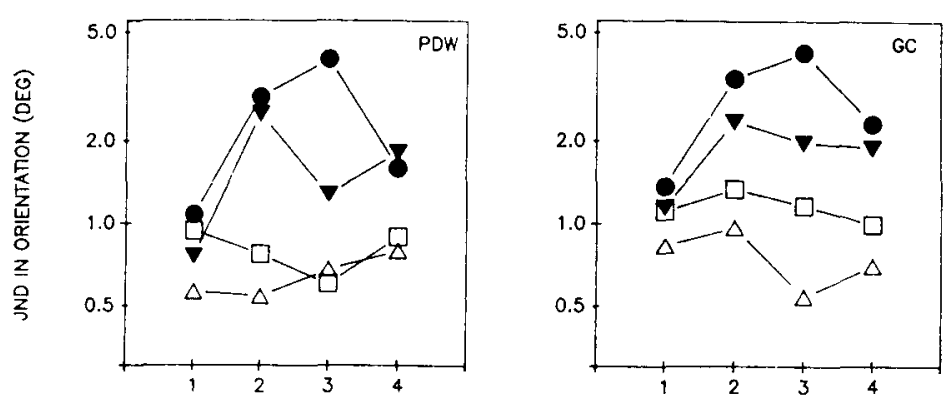

BACKGROUND CONDITION

B
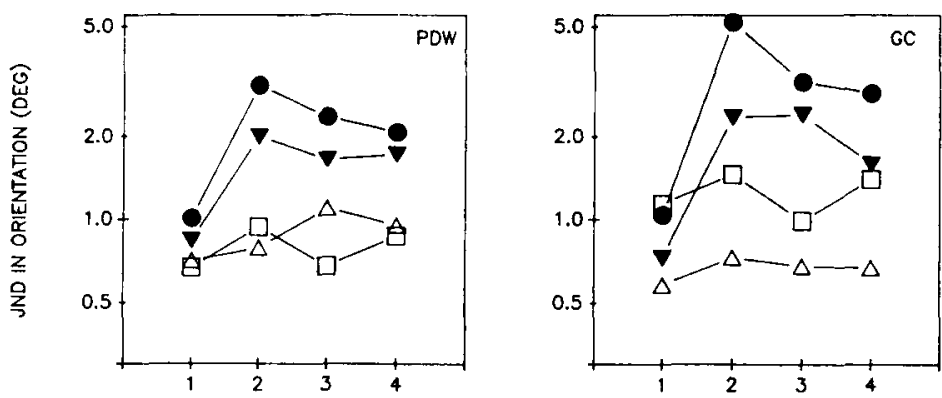

BACKGROUND CONDTION

C

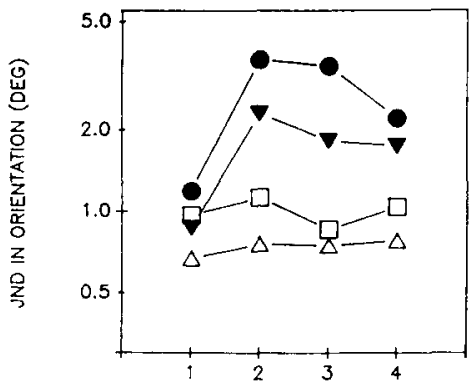

BACKGROUND CONDITION

Figure 5. JNDs in orientation measured for different contour types and plotted as a function of background condition for Subjects P.D.W. and G.C. Panel C presents average values, standard errors varying between $0.09^{\circ}$ and $0.75^{\circ}$. Solid dots refer to JNDs obtained with the standard stimulus pattern (see Figures 1A-1B). Solid, inverted triangles correspond to JNDs in orientation obtained with half the standard pattern (see Figures $4 A-4 B$ ). The open squares refer to JNDs measured after superimposing a line upon the occlusion-defined contour (see Figures 4C-4D). Finally, open upright triangles represent threshold values obtained with the dotted line (see Figures $4 E-4 F$ ). The texture backgrounds (Conditions 2,3 , and 4 ) represented in the two panels in row $A$ are line textures. The texture backgrounds represented in the two panels in row $B$ are annulus textures. 
(dotted line) and 1.13 (standard pattern). In Background Condition 2, JNDs increased to $3.64^{\circ}$ and $2.31^{\circ}$ for the standard pattern and its half version, respectively, whereas JNDs were $1.13^{\circ}$ for the superimposed solid line and $0.76^{\circ}$ for the dotted line. The average threshold values illustrate that the difference between occlusion- and non-occlusion-defined contours remained throughout Background Conditions 3 and 4 . Hence, the texture background effect cannot be demonstrated with any contour. Rather, this effect seems to be specifically linked with contours based on occlusion cues.

\section{GENERAL DISCUSSION}

Different ideas have been put forth about the way in which occlusion cues are interpreted by the visual system. The original cues-to-depth hypothesis advanced by Coren (1972) was essentially cognitive in nature and was related to other cognitive approaches such as the figural cue hypothesis (Gregory, 1972) and closure explanations (Osgood, 1953). Several authors (Brady \& Grimson, 1981; Marr, 1982), however, have argued that the logic of the cues-to-depth hypothesis might be built in at relatively low levels in the visual system. Indeed, the handling of contours generated by occlusion is an essential aspect of visual processing (Nakayama, Shimojo, \& Silverman, 1989; von der Heydt, 1987), and hence it could well be that special procedures are available to deal with this issue at early stages in the visual system. This position has gained weight after von der Heydt, Peterhans, and Baumgartner's (1984) discovery of units in monkey area V2 selective to the orientation of occlusion-defined (illusory) contours.

Peterhans et al. (1986) proposed a model of occlusiondefined contour perception that was based on grouping of the output of end-stopped cells. The end-stopped units would consist of a single excitatory subregion bordered at one end of its length axis by an inhibitory subregion. A row of such units, with the excitatory subregions all pointing in one direction, could detect the ends of lines coming in from the opposite direction. Grouping the output of such units could signal a contour with an orientation roughly orthogonal to the preferred orientation of the end-stopped cells. Contours induced by alternating opposite line ends, as in the stimulus used in the present study, would be encoded by an alternation of end-stopped cells with their excitatory subregion pointing to one or the other side. This is a simple model that can be used to explain a multitude of visual illusions (von der Heydt \& Peterhans, 1989a). The essential feature of this model is that it is local. Contours defined by occlusion-in artificial stimulus patterns and in the real life environmentwould be detected through local analysis of line ends.

The present study shows that JNDs in orientation increase when an occlusion-defined contour stimulus is presented on a background texture. JNDs increased by a factor of nearly 3 , even after removal of texture elements near the inducing endpoints. The fact that the increase remained significant in a condition in which almost half of the illusory contour pattern was free of texture (Background 4) suggests that this effect was not caused purely by disturbing a local mechanism processing line ends. Indeed, the presence of texture elements between the inducing elements creates the impression that the surface at each side of the contour is transparent and thereby corrupts the interpretation of the displaced sets of circle halves as belonging to occluding surfaces. The fact that the effect of the background texture did not depend on the type of texture, and that it was present only for contours defined by occlusion cues, strongly supports the idea that the threshold increases observed in all texture background conditions were to a significant extent a consequence of the reduced strength of occlusion cues. Hence, we suggest that the mechanism interpreting line ends in artificial 2-D patterns as occlusion cues contributes to the accuracy with which an observer perceives the contours defined by these line ends.

Interestingly, thresholds obtained with dotted lines, made of the dots corresponding to the ends of inducing lines, were significantly smaller than those obtained with the standard occlusion-defined contour (Experiment 3). This difference cannot be explained with Peterhans et al.'s (1986) model. Physiological (von der Heydt, 1987; von der Heydt \& Peterhans, 1989b) as well as psychophysical evidence (Kennedy, 1978) suggests that the end-stopped units detecting the line ends are orientation selective. Therefore, a small unoriented dot is likely to be a less effective stimulus than is an optimally oriented line end, and hence thresholds are expected to increase in size, rather than decrease. The decrease that we observed could reflect the contribution of low-pass filtering mechanisms that could infer orientation from the juxtaposition of the dots. Alternatively, the decrease could have been due to signals from small nonoriented cells, which might form a better input to the contour orientation discrimination mechanism than does the end-stopped cell input.

The changes in accuracy of orientation judgments in texture background conditions are quantitative changes, which have to be distinguished from qualitative changes such as changes in the subjective quality of illusory contours. Manipulations of transparency have previously been reported to destroy the perception of illusory contours (Rock \& Anson, 1979). Another visual illusion known to be affected by manipulations of transparency is stereo capture (Ramachandran \& Cavanagh, 1985; Nakayama, Shimojo, \& Ramachandran, 1990). Furthermore, occlusion and transparency cues are highly relevant for the solution one gives to the aperture problem for motion (Shimojo, Silverman, \& Nakayama, 1989; Vallortigara \& Bressan, 1991; Watanabe \& Cavanagh, 1991). All these studies demonstrate the close intertwining between depth and occlusion cues, transparency and the perception of illusory contours (Nakayama et al., 1989).

The subjects in the present study reported that although the endpoints along the contour remained clearly visible, the subjective impression of a contour linking the endpoints was strongly reduced when the texture was intro- 
duced. Furthermore, except for Subject H.K., the subjects reported seeing the texture behind the inducing circle halves. H.K. reported an ambiguous percept, not being able to define the texture as a background or a foreground. Hence, it seems that reducing the strength of the occlusion cues defining a contour, by creating the impression of transparency, not only produces quantitative changes in the perception of that contour but also reduces the illusory quality of the occlusion-defined contour. One could argue that the direct cause leading to increased orientation thresholds is the loss of the illusory appearance of the contour. This idea agrees with our finding that the background texture does not raise orientation thresholds if contours are used that do not generate an illusory boundary coinciding with the contour.

Our finding of a measurable contribution of occlusion cues to the accuracy of contour perception in artificial 2-D patterns supports Peterhans et al.'s (1986); and von der Heydt's (1987) broader claim that the mechanism responsible for illusory contour perception plays an important role in the recovery of occlusion-defined edges from reallife visual scenes. However, the data also suggest that their local model may be an insufficient basis for such a mechanism. Indeed, their model leaves no room for disambiguating the significance of line ends. Even if one accepts that the end-stopped cells are disparity sensitive (von der Heydt \& Peterhans, 1989b), there will be a number of instances in which these units are stimulated by line ends that are not related to a contour (e.g., when they belong to isolated small objects dispersed in the environment). Clearly, potential occlusion cues should only give rise to contour perception if there is evidence that these cues indicate borders of solid surfaces. Thus, some analysis of surfaces must precede or at least should accompany the analysis of occlusion cues. This constraint is taken into account by computational theories of vision (Brady \& Grimson, 1981; Buckley, Frisby, \& Mayhew, 1989; Marr, 1982). In these theories, contours are constructed during the analysis of relative depth between surfaces, rather than after the analysis of isolated endpoints. In that view, it can easily be understood that any manipulation reducing the percept of certain elements belonging to occluding surfaces also diminishes the strength of the potential contour seen at the border between these surfaces.

If one accepts that a local model is insufficient to explain illusory contour perception, one can speculate that the locus of the processes generating such contours may not be limited to area V2, which is where von der Heydt et al. (1984) found cells responsive to illusory contours. Our results suggest that an analysis of relative depth between surfaces and transparency of surfaces contributes to illusory contour perception. Assuming that this analy. sis does not take place in area V2, one could propose that the responses to illusory contours observed in single cells in area $\mathrm{V} 2$ in fact are generated in higher order areas and then backprojected to area V2. Alternatively, it is possible that illusory contours are generated by local encoding of line ends at the level of area V2, as suggested by
Peterhans et al. (1986), but that the outcome of that processing stage is modified by an analysis of surfaces taking place in higher order areas (e.g., area V3 or V4). The presence of visual illusions in 2-D patterns may depend on the outcome of that analysis. In both scenarios, feedback is an essential feature. The usefulness of feedback in the processing of illusory contours has been convincingly demonstrated in a simulation study of Finkel and Edelman (1989). Also, Nakayama et al. (1990) suggested that the modification of the perception of illusory contours through manipulations of transparency might be mediated by feedback connections. Clearly, the abundance and strength of feedback in the visual system agrees with such proposals (Desimone \& Ungerleider, 1989; Ungerleider \& Mishkin, 1982). The fundamental issue, however, of the functional significance of feedback connections in the perception of illusory contours, and in perception in general, remains unresolved.

\section{REFERENCES}

Anderson, G. J., \& Cortese, J. M. (1989). 2-D contour perception resulting from kinetic occlusion. Perception \& Psychophysics, 46, 49-55.

Baumgartner, G., von der Heydt, R., \& Peterhans, E. (1984). Anomalous contours: A tool in studying the neurophysiology of vision. Experimental Brain Research, 9(Suppl.), 413-419.

Brady, M., \& Grimson, W. E. I. (1981). The perception of subjective surfaces (Artificial Intelligence Memo No. 666). Cambridge: Massachusetts Institute of Technology, AI Laboratory.

Bruno, N., \& Bertamini, M. (1990). Identifying contours from occlusion events. Perception \& Psychophysics, 48, 331-342.

Buckley, D., Frisby, J. P., \& Mayhew, J. E. W. (1989). Integration of stereo and texture cues in the formation of discontinuities during three-dimensional surface interpolation. Perception, 18, 563-588.

Coren, S. (1972). Subjective contours and apparent depth. Psychological Review, 70, 359-367.

Coren, S., \& Girgus, J. S. (1978). Seeing is deceiving: The psychology of visual illusions. Hillsdale, NJ: Erlbaum.

Desimone, R., \& UNGERLEIDER, L. G. (1989). Neural mechanisms of visual processing in monkeys. In F. Boller \& J. Grafman (Eds.), Handbook of neuropsychology (Vol. 2, pp. 267-299). Amsterdam: Elsevier.

FINKEL, L. H., \& EDELMAN, G. M. (1989). Integration of distributed cortical systems by reentry: A computer simulation of interactive functionally segregated visual areas. Journal of Neuroscience, $\mathbf{9}$, 3188-3208.

Gregory, R. L. (1972). Cognitive contours. Nature, 238, 51-52.

HALPERN, D. F. (1981). The determinants of illusory contour perception. Perception, 10, 199-213.

HALPERN, D. F. (1983). The multiple determination of illusory contours: 1. A review. Perception, 12, 281-291.

KANISZA, G. (1955). Margini quasi-percettivi in campi con stimulazioni omogenea [Conditions and effects of apparent transparency]. Rivista di Psicologia, 49, 7-30.

Kennedy, J. M. (1978). Illusory contours and the ends of lines. Perception, 7, 605-607.

MARR, D. (1982). Vision: A computational investigation into the human representation and processing of visual information. San Francisco: W. H. Freeman.

Metelli, F. (1974a). Achromatic color conditions in the perception of transparency. In R. B. MacLeod \& H. L. Pick (Eds.), Perception: Essays in honor of J. J. Gibson (pp. 95-116). Ithaca, NY: Cornell University Press.

Metelli, F. (1974b, April). The perception of transparency. Scientific American, pp. 90-98.

Mustillo, P., Francis, E., Oross, S., III, Fox, R., \& Orban, G. A. 
(1990). Anisotropies in global stereoscopic orientation discrimination. Vision Research, 28, 1315-1321.

Nakayama, K., Shimojo, S., \& Ramachandran, V. S. (1990). Transparency: Relation to depth, subjective contours, luminance, and neon color spreading. Perception, 19, 497-513.

Nakayama, K., Shimojo, S., \& Silverman, G. H. (1989). Stereoscopic depth: Its relation to image segmentation, grouping, and the recognition of occluded objects. Perception, 18, 55-68.

Orban, G. A., Vandenbussche, E., \& Vogels, R. (1984). Human orientation discrimination tested with long stimuli. Vision Research, 24, $121-128$.

OsGood, C. E. (1953). Method and theory in experimental psychology. New York: Oxford University Press.

PARks, T. E. (1984). Illusory figures: A (mostly) atheoretical review. Psychological Bulletin, 95, 282-300.

Peterhans, E., von Der Heydr, R., \& Baumgartner, G. (1984). Illusory contour stimuli reveal stages in visual cortical processing. Perception, 13, A16-A17. (Abstract)

Peterhans, E., von der Heydt, R., \& Baumgartner, G. (1986). Neuronal responses to illusory contour stimuli reveal stages of visual cortical processing. In J. D. Pettigrew, K. J. Sanderson, \& W. R. Levick (Eds.), Visual neuroscience (pp. 343-351). Cambridge: Cambridge University Press.

PORAC, C. (1978). Depth in objective and subjective contour patterns. Bulletin of the Psychonomic Society, 11, 103-105.

PruTChaRD, W. S., \& W ARMS, J. S. (1983). Attentional processing and the subjective contour illusion. Journal of Experimental Psychology: General, 112, 145-175.

Ramachandran, V. S., \& Cavanagh, P. (1985). Subjective contours capture stereopsis. Nature, 317, 527-530.

REYNOLDS, R. I. (1981). Perception of an illusory contour as a function of processing time. Perception, 10, 107-115.

Rock, I., \& ANSON, R. (1979). Illusory contours as a solution to a problem. Perception, 8, 665-681.

Rubin, E. (1958). Figure and ground. In D. C. Beardslee \& M. Wertheimer (Eds.), Readings in perception (pp. 194-203). Princeton, NJ: Van Nostrand

SchumanN, F. (1900). Beiträge sur Analyse der Gesichtswahrnehmungen. Zeitschrift für Psychologie, 23, 1-32.

ShANK, M. D., \& WALKER, J. T. (1989). Figure-ground organization in real and subjective contours: A new ambiguous figure, some novel measures of ambiguity, and apparent distance across regions of figure and ground. Perception \& Psychophysics, 46, 127-138.

Shimojo, S., Silverman, G. H., \& Nakayama, K. (1989). Occlusion and the solution to the aperture problem for motion. Vision Research, 29, 619-626.

Ungerleider, J. G., \& Mishkin, M. (1982). Two cortical visual systems. In D. J. Ingle, M. A. Goodale, \& R. J. W. Mansfield (Eds.), Analysis of visual behavior (pp. 549-586). Cambridge, MA: MIT Press.

Vallortigara, G., \& Bressan, P. (1991). Occlusion and the perception of coherent motion. Vision Research, 31, 1967-1978.

Vogels, R., \& Orban, G. A. (1985). The effect of practice on the oblique effect in line orientation judgments. Vision Research, 25 , 1679-1687.

Vogels, R., Orban, G. A. (1986). Decision processes in visual discrimination of line orientation. Journal of Experimental Psychology: Human Perception \& Performance, 12, 115-132.

VoGels, R., Orban, G. A. (1987). Illusory contour orientation discrimination. Vision Research, 27, 453-467.

VON DER HEYDT, R. (1987). Approaches to visual cortical function. Reviews in Physiology, Biochemistry \& Pharmacology, 108, 69-150. von der Heydt, R., \& Peterhans, E. (1989a). Ehrenstein and Zöllner illusions in a neuronal theory of contour processing. In $C$. $M$. Dickinson \& J. J. Kulikowski (Eds.), Seeing contour and colour: Proceedings of the Third International Symposium of the Northern Eye Institute (pp. 729-734). London: Pergamon.

von der Heydt, R., \& Peterhans, E. (1989b). Mechanisms of contour perception in monkey visual cortex: I. Lines of pattern discontinuity. Journal of Neuroscience, 9, 1731-1748.

von der Heydt, R., Peterhans, E., \& Baumgartner, G. (1984). Illusory contours and cortical neuron responses. Science, 224, $1260-1262$

Watanabe, T., \& Cavanagh, P. (1991). Texture and motion spreading, the aperture problem, and transparency. Perception \& Psychophysics, 50, 459-464.

WeTHerill, G. B., \& LeVITT, H. (1965). Sequential estimation of points on a psychometrical function. British Journal of Mathematical \& Statistical Psychology, 18, 1-10.

(Manuscript received December 21, 1992; revision accepted for publication June 17, 1993.) 